\title{
Management of orbital injury and expanding orbital haematoma
}

BRIDGET HEMMANT AND CARL PHILPOTT

\author{
Definition \\ Causes \\ Spontaneous \\ Head/facial trauma \\ latrogenic \\ Diagnosis \\ Management \\ Spontaneous
}

\section{DEFINITION}

Expanding orbital haematoma (or retrobulbar haemorrhage) is a rare, potentially sightthreatening complication which results from trauma to one of the orbital arteries - namely, the branches of the ophthalmic artery (anterior and posterior ethmoidal arteries) or orbital vein. Such an orbital injury can result from head trauma or can be iatrogenic (e.g. following injection into the orbit or surgery). Very rarely, it can occur spontaneously. Visual loss results either from central retinal artery occlusion, optic neuropathy from stretching of the optic nerve, or direct compression of the optic nerve venous drainage. As the haematoma expands within the confines of the bony orbit, the tense sheet of tissue that stretches across the anterior orbital opening - the orbital septum (Figures 5.1 and 5.2) - prevents the escape of blood. This leads to a rise in intraorbital pressure and visual

\author{
Head/facial trauma $\quad 42$ \\ latrogenic $\quad 42$ \\ Surgical approach for retrobulbar haemorrhage 43 \\ Management of other orbital complications \\ of iatrogenic orbital trauma $\quad 43$ \\ Key learning points $\quad 44$ \\ References 44 \\ Further reading $\quad 45$
}

loss. Prompt therapeutic intervention, including an emergency lateral canthotomy and cantholysis and/or orbital decompression, may restore vision.

\section{CAUSES}

Retrobulbar haemorrhage (RBH) can occur as a result of trauma, eyelid or orbital surgery, and other processes affecting the circulation within the orbit, such as arteriovenous malformations and lymphangiomas. The use of anticoagulant medication can increase the risk of $\mathrm{RBH}$ after relatively minor head trauma.

Although uncommon, there is an association between functional endoscopic sinus surgery (FESS) and orbital complications such as $\mathrm{RBH}$, and if the orbit is inadvertently entered surgically, injury to other structures in the orbit can occur. 


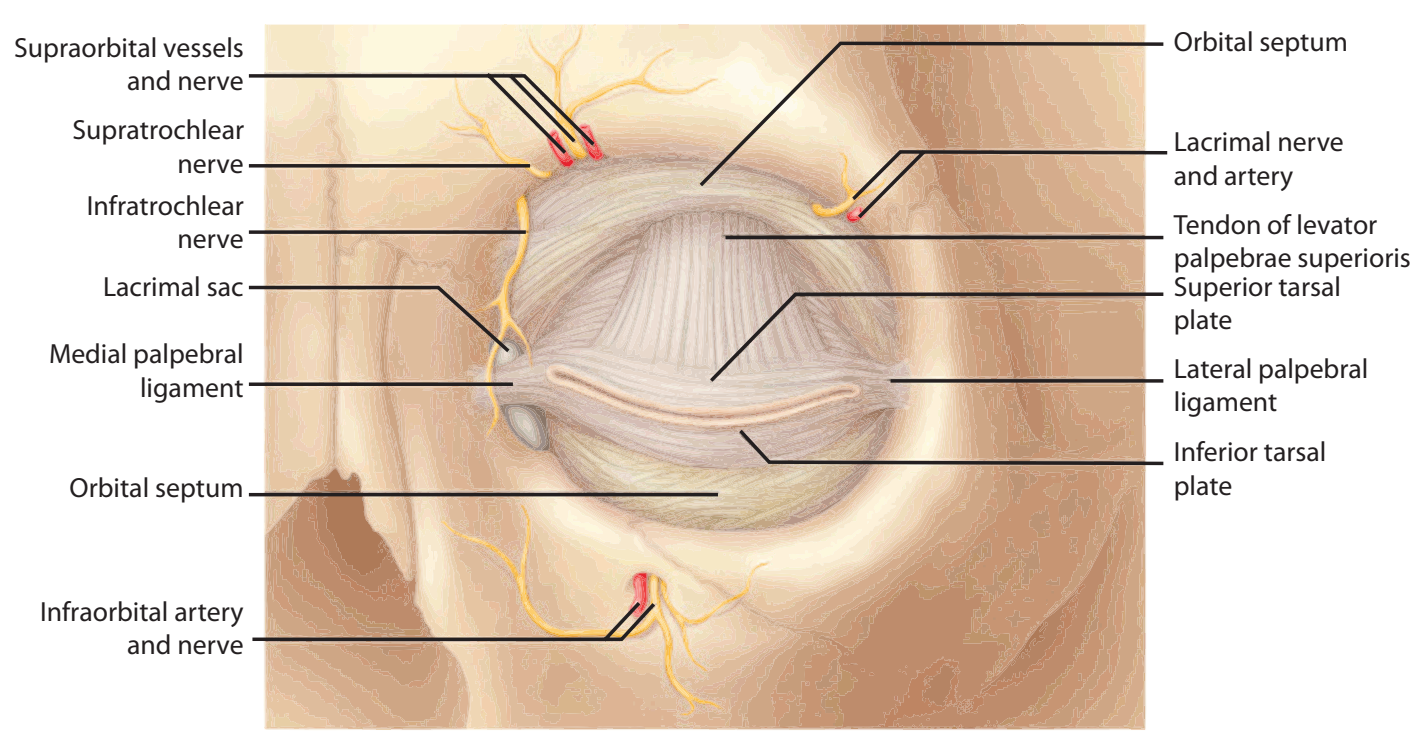

Figure 5.1 Anterior view of the orbit with orbital septum in place - dividing the anterior septal structures from the orbital contents.

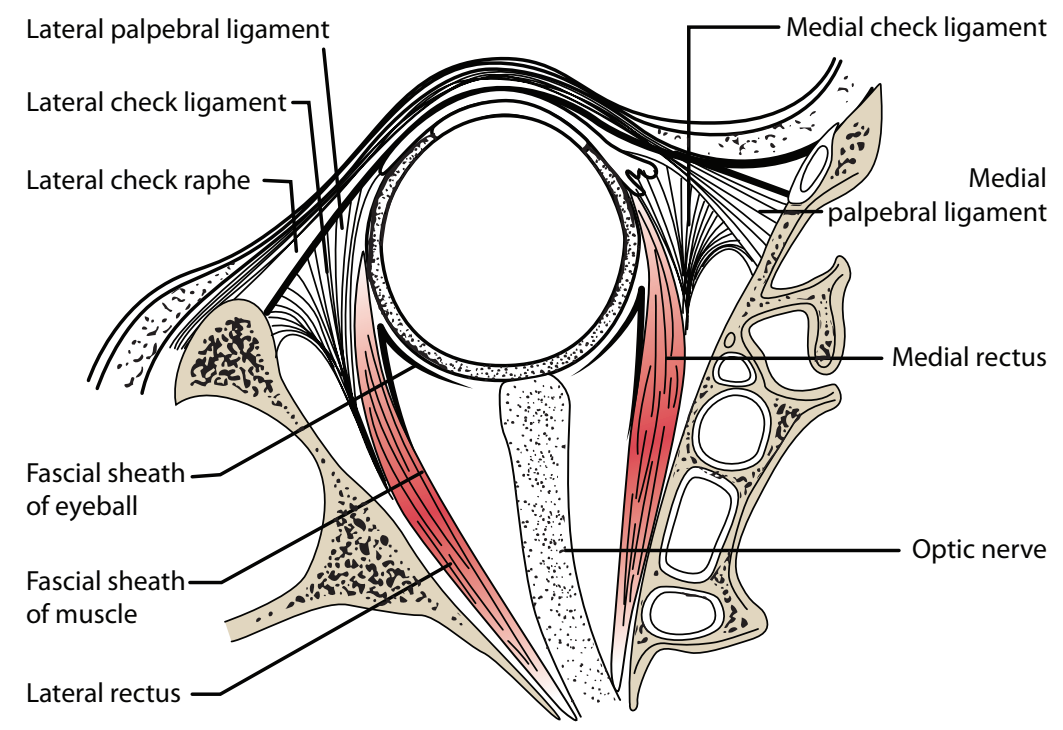

Figure 5.2 Anterior posterior cross section of the orbit, demonstrating the anatomy of the lateral canthal ligaments (lateral palpebral raphe, lateral check ligament and lateral palpebral ligament) attaching the orbital septum to the orbital rim. 
The other structures at risk from direct trauma are the optic nerve and the extraocular muscles, particularly the medial recti muscles. The rise in the popular use of microdebriders ${ }^{1}$ has also seen a rise in debrider related injuries; if the surgeon is not alert to the presence of periorbital fat in the field during FESS, then it can be very easy to quickly find both fat and muscle from the recti being removed by the debrider. Damage to the optic nerve can lead to irreversible visual loss, and damage to the muscles can cause diplopia (double vision). There is also potential risk to the lacrimal drainage system during FESS surgery. This results in epiphora (watery eye); while this can transiently affect the vision, the tear duct obstruction can usually be corrected surgically with a dacryocystorhinostomy procedure (DCR) at a later date, should the watering not resolve (Figure 5.3).

\section{SPONTANEOUS}

With spontaneous retrobulbar haemorrhage (SRH), the patient will normally complain of a rapid acute onset proptosis (forward bulging of the globe), likely associated with some pain and loss of vision in the affected eye. There may also be an acute onset diplopia. If the haematoma is small, there may be mild optic neuropathy with a subtle change in the colour vision prior to any visual loss. SRH is more likely to occur in patients with pre-existing orbital vascular malformations, such as orbital lymphangioma, and arteriovenous malformations. Blood dyscrasias (e.g. sickle anaemia) are another rare cause of spontaneous $\mathrm{RBH}$.

\section{HEAD/FACIAL TRAUMA}

Retrobulbar haemorrhage as a result of head or facial trauma may occur if there is trauma, either direct (via a penetrating orbital injury) or via a shearing action to the ophthalmic artery and/or the posterior and anterior ethmoidal arteries. The ethmoid arteries branch from the ophthalmic artery as it passes along the medial orbital wall at an angle of $90^{\circ}$ and pass through fenestra in the lamina papyracae, placing them at risk from shearing forces. Patients on anticoagulants are at greater risk from $\mathrm{RBH}$, which can occur after relatively minor head trauma (Figure 5.4).

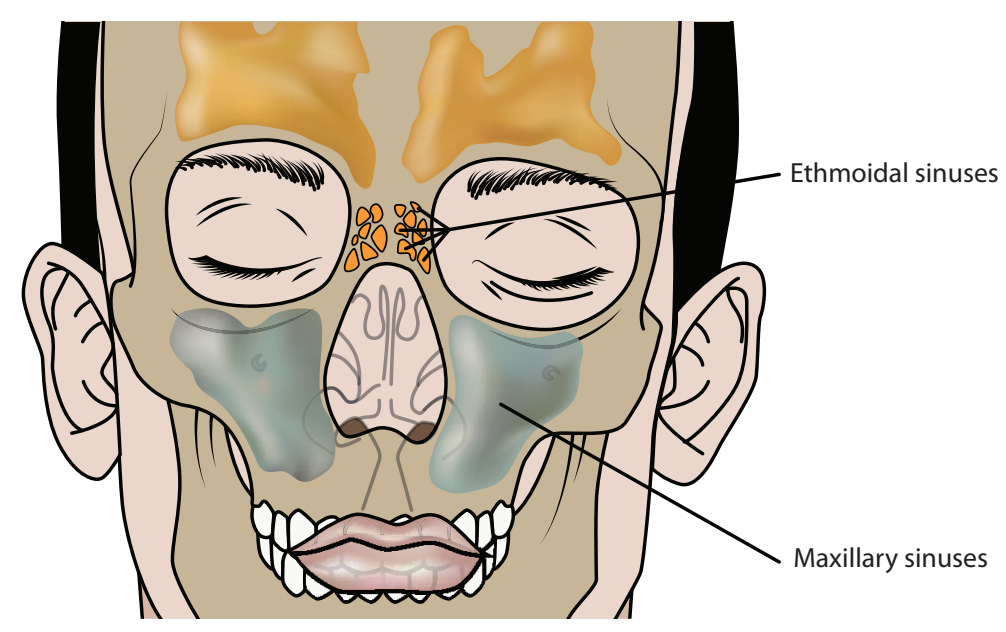

Figure 5.3 Demonstrates the anatomy of the orbit and the proximity of the ethmoid sinuses to the orbit just separated by the thin lamina papyracae. 


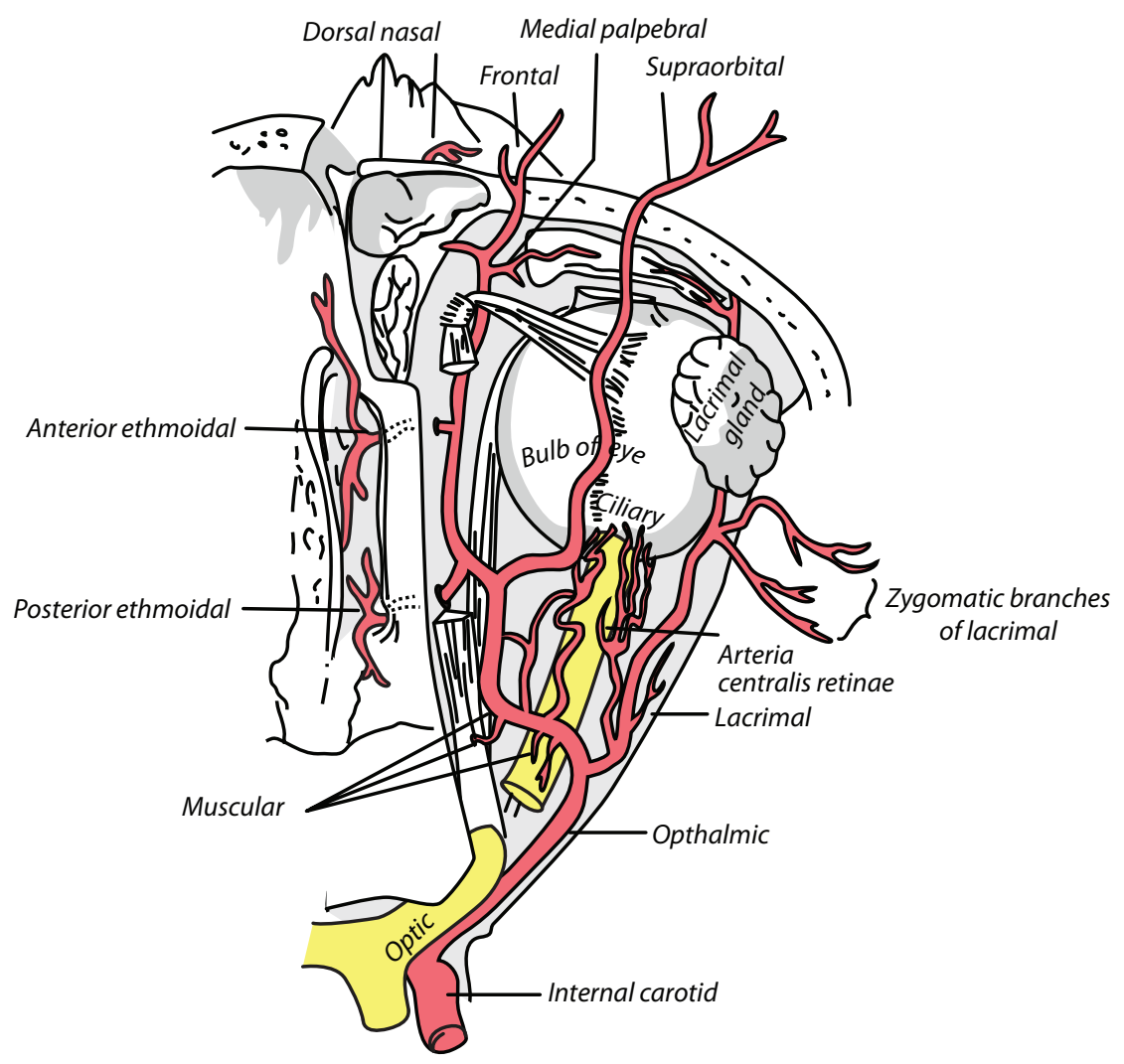

Figure 5.4 The vascular anatomy of the orbit and the passage of the anterior and posterior ethmoidal arteries through the lamina papyracae.

\section{IATROGENIC}

Any surgery (or injection) which encroaches on the orbit and its contents within the post-septal space (posterior to the orbital septum) has the risk of causing a retrobulbar haemorrhage. Retrobulbar injections were first introduced in the late nineteenth century and have commonly been given for cataract and other intraocular surgery (during the latter part of the twentieth century) with the attendant risk of $\mathrm{RBH}$ and trauma to the optic nerve. These injections were superseded by periorbital injections, and subsequently by topical anaesthesia, where there is no such risk. Surgery that penetrates the orbital septum anteriorly or the lamina papyracae medially carries the risk of damage to the orbital vessels. In ENT surgery and endoscopic sinus surgery, in particular, there is a risk that the thin lamina papyracae may be breached and the orbit entered. The risk of orbital injury varies widely but is in the vicinity of $0.06-2.25 \%$. The microdebrider is the instrument most likely to be associated with this type of surgical trauma. Preoperative CT scans of the orbit and sinuses can aid the pre-operative plan by better visualisation of the ethmoidal anatomy. A pre-operative check of the scans should aim to identify the following areas for the risk of potential injury or complication ${ }^{8}$ :

- Anterior and posterior ethmoid arteries - these can be seen in a coronal plane (Figure 5.5) and most often will lie within the skull base; however, in a minority of cases, the anterior ethmoid artery may lie within a bony 'mesentery' beneath the skull base, where it is at greater risk of injury from through-cutting instruments.

- Aerated posterior spheno-ethmoidal cells (Onodi cells) may lie alongside the optic nerve canal and may place a greater risk of injury 


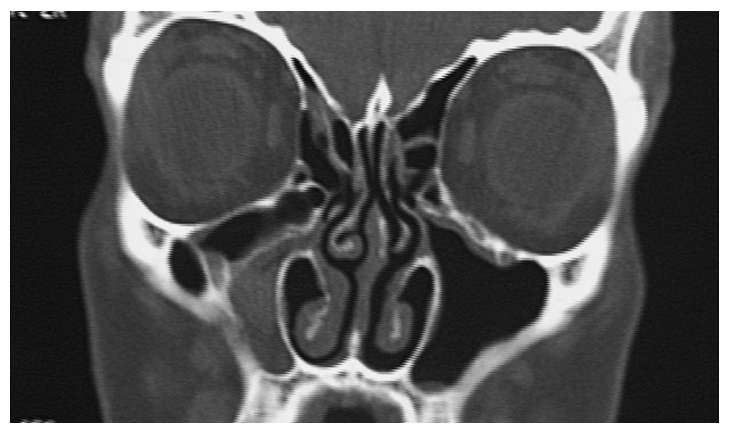

Figure 5.5 Showing the anterior ethmoid artery (also known as Kennedy's nipple) on a coronal CT scan slice and an infra-orbital ethmoid cell. (NB need to find a better CT)

during ethmoidectomy/sphenoidotomy, especially if not recognised beforehand, as the bony covering of the nerve may be thin or even deficient (Figure 5.6). These anatomical variants are more common in some ethnic groups, e.g. South East Asians.

- Dehiscence of the lamina papryacea can be identified on coronal and axial CT sequences, and in cases of revision FESS, close attention should be paid to the possibility of this having occurred previously, especially where polyps are present in the ethmoidal areas.

- Hypoplasia of the maxillary sinus may affect the relative position of the lamina to the medial wall of the maxilla, with the possibility of the lamina lying more medial in the nose than

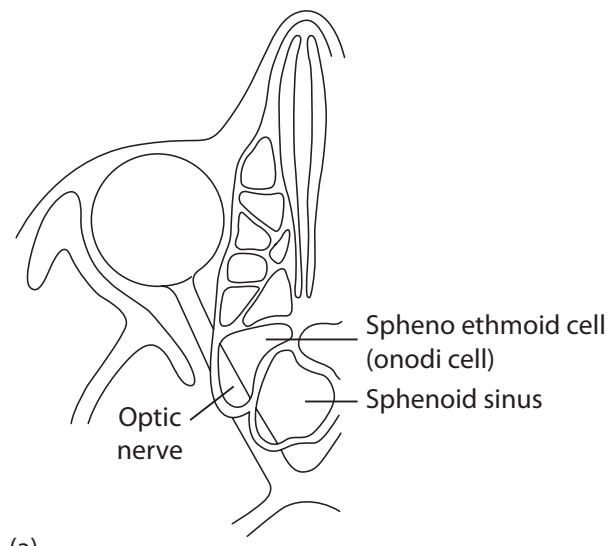

(a)

Figure 5.6 (a) Onodi cell. (b) Endoscopic view of optic nerve in Onodi cell. the maxillary wall and leading the surgeon to believe they are entering the anterior ethmoids when in fact they are breaching the lamina.

- Infra-orbital ethmoid (Haller) cells are best identified on the coronal CT planes and should be identified if entry to the maxillary sinus and ethmoid infundibulum is anticipated during surgery (Figure 5.5).

\section{DIAGNOSIS}

Signs of a retrobulbar haemorrhage include periorbital swelling, bruising and oedema, conjunctival chemosis (fluid under the conjunctiva) and subconjunctival haemorrhage (Figure 5.7). The

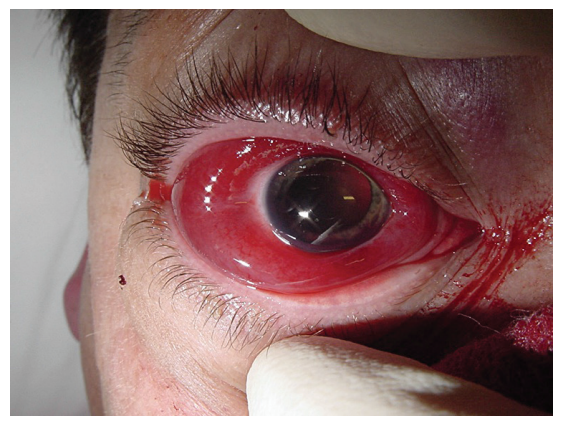

Figure 5.7 Clinical image of a patient's right eye with subconjunctival haemorrhage and chemosis.

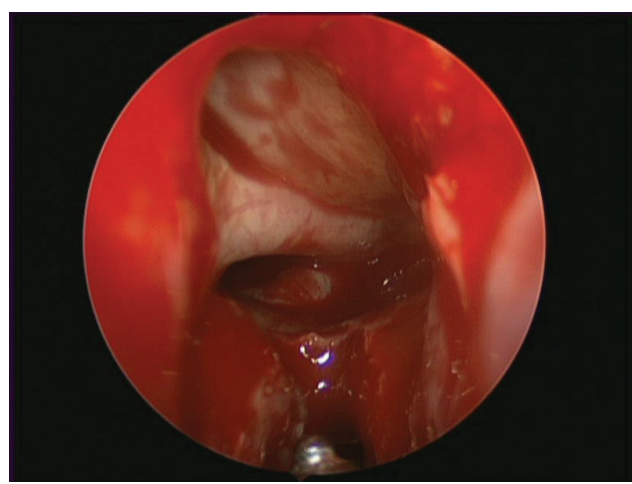

(b) 
orbit may feel tense and there may be proptosis (bulging forwards of the globe). Signs of optic nerve compromise include dilation of the pupil with a relative afferent pupillary defect (RAPD). If the patient is awake and cooperative, the visual function can be assessed further to check for optic neuropathy by assessing the colour vision and the visual field. Ocular motility can also be assessed to look for muscle restriction or weakness plus diplopia. During FESS it is advisable to keep the eyes uncovered to monitor them for any movement from traction on the orbital fat. Other indications of orbital penetration during the surgery are sudden swelling and bruising of the eyelids, conjunctival chemosis, subconjunctival haemorrhage and rapidly increasing proptosis. A more rapid onset follows an arterial bleed, which can result if a transected ethmoidal artery retracts into the orbit and continues to bleed. Injury to the optic nerve may be suspected if the pupil suddenly dilates during surgery or if, following surgery, there is visual loss and poorly reactive pupils with a relative afferent pupil defect. The dilation may be due to globe ischaemia or damage to the pupillomotor nerve fibres.

If an $\mathrm{RBH}$ is suspected, there is no time to order tests such as CT/MRI scans, as emergency treatment, either with a lateral canthotomy and cantholysis and/or with an orbital decompression, is required to drain the haematoma collection. However, if there is no $\mathrm{RBH}$ but direct damage to the optic nerve is suspected, an MRI of the orbit to visualise the soft tissues and bone is helpful with onward referral to an ophthalmic surgeon.

If there is diplopia following FESS surgery and there is a defect in the ocular motility that was not present prior to surgery, damage to the extraocular muscles should be assessed. Imaging with a CT or MRI can be helpful. If there is muscle or soft tissue entrapment in the orbital wall, this should be dealt with by an orbital surgeon before any strabismus surgery is considered. Patients may complain of epiphora following FESS surgery. This may be due to post-operative oedema in the area of the lacrimal drainage system, and this does often settle with reassurance and time. Radiology imaging may be helpful if the epiphora does not settle within 4-6 weeks.

\section{MANAGEMENT}

\section{SPONTANEOUS}

Whenever a retrobulbar haemorrhage is suspected, this is an ophthalmic emergency, and decompression of the orbit should be considered on an urgent basis. If there is a clotting disorder or blood dyscrasia clearly this needs to be addressed in addition to involvement of the haematology team. Depending on the optic nerve function, there may be time to consider an urgent radiology image (CT or MRI) to look for the source of the bleed.

\section{HEAD/FACIAL TRAUMA}

Patients with head and facial trauma significant enough to cause a retrobulbar haemorrhage may have other significant injuries that are immediately life-threatening. These need to be prioritised. There is, however, a significant risk of visual loss where there is an expanding orbital haematoma. If there is a rapidly progressive proptosis with visual loss and the history is suggestive of a retrobulbar haematoma, it may be necessary to perform an emergency lateral canthotomy and cantholysis, as delay can lead to permanent visual loss. If the diagnosis is less certain and where other injuries are suspected, then an urgent orbital CT or MRI should be performed. If an intraorbital foreign body is suspected, the nature of the material may dictate what type of imaging is ordered. This should be discussed with the radiologist. These patients should be referred to the ophthalmology department, preferably to a surgeon experienced in orbital surgery.

\section{IATROGENIC}

If a retrobulbar haemorrhage is suspected during a procedure, the surgery should cease immediately. If the haemorrhage occurs during FESS surgery, packing and suction should be removed from the site; the surgeon should not be tempted to continue to use the microdebrider to remove fat to 'decompress' the orbit. All ENT surgeons who undertake FESS should be familiar with the emergency procedure of lateral canthotomy and cantholysis, which can be practised in cadaveric sinus courses, but can also 
be observed in ophthalmology lists where entropion surgery is undertaken. Whilst the latter is an elective setting without the concern of a proptosing globe, it enables clear visualisation of the lateral canthal tendon that is superior to cadaveric specimens.

\section{SURGICAL APPROACH FOR RETROBULBAR HAEMORRHAGE}

Lateral canthotomy (Figures 5.8 and 5.9): A pair of sharp straight scissors are used to make a cut in the lateral canthal angle (canthotomy), and then the scissors are rotated $90^{\circ}$ to cut through the lateral canthal tendon (lateral cantholysis) at its attachment to the bony orbital rim. The third cut is to cut deep to the skin, but through the orbital septum and conjunctiva below the tarsal plate in order to allow the haematoma to drain (Figure 5.9). In addition, the orbital pressure should be reduced. The patient should be given mannitol $-1 \mathrm{gm} / \mathrm{kg}$ IV over 30 minutes - plus acetazolamide - $500 \mathrm{mg}$ IV. An urgent ophthalmology review should be arranged and an external (or endoscopic) orbital decompression considered as the next immediate intervention, depending upon effectiveness of the canthotomy. As visual loss is the main risk with a retrobulbar haemorrhage, reducing the pressure in the orbit and allowing the haematoma to evacuate are the main concerns. Cosmesis is a secondary issue, as eyelid reconstruction is possible at a later date if required.

Q2 Table 5.1 Steps involved in managing a peroperative retrobulbar haemorrhage

\begin{tabular}{ll} 
Step & $\begin{array}{c}\text { Management of retrobulbar } \\
\text { haemorrhage in FES surgery }\end{array}$ \\
\hline 1 & - Remove packing and suction at \\
bleeding site if occurs during FESS \\
2 & - Lateral canthotomy and cantholysis \\
3 & - Mannitol - $1 \mathrm{gm} / \mathrm{kg} \mathrm{IV} \mathrm{over} 30$ \\
& minutesSlb \\
4 & - Acetazolamide - 500 mg IV \\
5 & - Urgent ophthalmology review \\
6 & - Consider orbital decompression \\
& (externally or endoscopically) \\
\hline
\end{tabular}
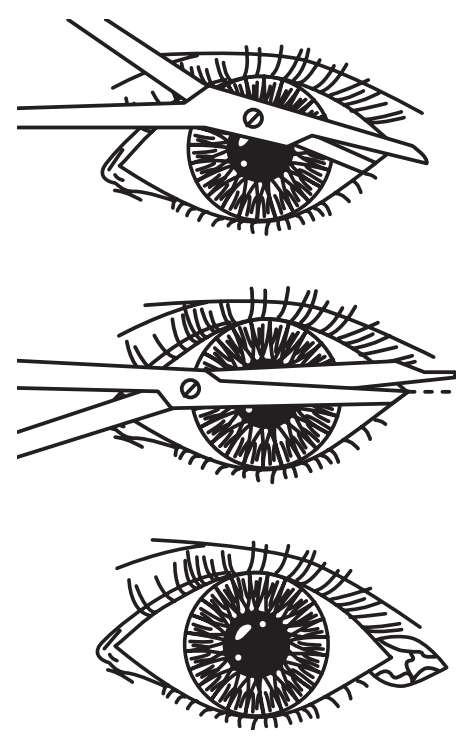

Figure 5.8 Lateral canthotomy to release the lateral canthal angle.

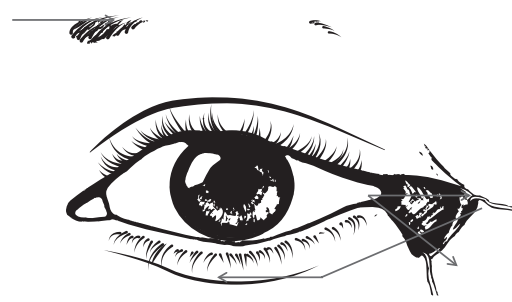

Figure 5.9 Diagram showing the incisions in blue to open the lateral canthal angle and to cut the lateral canthal tendon. The line in orange demonstrates the further incision - through the lid tissues posterior/deep to the skin below the lower border of the tarsal plate and through the orbital septum to release the septum and allow the haematoma to drain.

\section{MANAGEMENT OF OTHER ORBITAL COMPLICATIONS OF IATROGENIC ORBITAL TRAUMA}

FESS in inexperienced hands may be associated with RBH and with other complications, including direct trauma to the optic nerve, to the extraocular muscles and to the lacrimal drainage apparatus. 
If following FESS a patient complains of visual impairment by an ophthalmic surgeon is required. If direct damage to the optic nerve is suspected, an MRI of the orbit to visualise the soft tissues and bone is helpful.

If there is diplopia following FESS surgery, assessment by an ophthalmic surgeon is required. If there is a defect in the ocular motility that was not present prior to surgery, damage to the extraocular muscles should be considered. Imaging with a CT or MRI can be helpful. If there is muscle or soft tissue entrapment in the orbital wall or enophthalmos (sunken eye), this should be dealt with by an orbital surgeon before any strabismus surgery is considered.

Patients may complain of epiphora following FESS surgery. This may be due to post-operative oedema in the area of the lacrimal drainage system, and this does often settle with reassurance

\section{KEY LEARNING POINTS}

- Retrobulbar haemorrhage is an ophthalmic emergency. Visual loss is the main risk and may be permanent if timely treatment is not administered.

- FESS may be associated with orbital trauma, and $\mathrm{RBH}$ in particular, when the surgeon is inexperienced and is operating in the area of the ethmoid sinus.

- Progressive proptosis associated with periorbital bruising and swelling with a tense orbit should be assumed to be a retrobulbar haemorrhage until proven otherwise.

- In an acute setting per-operatively (e.g. during FESS), surgery should immediately cease, and a lateral canthotomy and cantholysis should be performed and the haematoma encouraged to drain.

- Intravenous mannitol and acetazolamide should be administered to lower the orbital pressure osmotically.
- Emergency referral to ophthalmology should be made.

- The pupillary responses and vision should be monitored post canthotomy and a formal external orbital decompression considered.

- As visual loss is the main risk with a retrobulbar haemorrhage, reducing the pressure in the orbit and allowing the haematoma to evacuate are the main concerns. Cosmesis is secondary, with later reconstruction being possible if required.

and time. Radiology imaging may be helpful if the epiphora does not settle within 4-6 weeks. If after 4-6 months the epiphora has not resolved and if there is a non-patent lacrimal drainage system, a lacrimal bypass procedure such as a dacryocystorhinostomy (DCR) can be considered.

\section{REFERENCES}

1. Bruggers S, Sindwani R. Evolving trends in powered endoscopic sinus surgery. Otolaryngol Clin North Am 2009; 42(5): 789-98, viii.

2. Bhatti MT, Giannoni CM, Raynor E, Monshizadeh R, Levine LM. Ocular motility complications after endoscopic sinus surgery with powered cutting instruments. Otolaryngol Head Neck Surg 2001; 125(5): 501-9.

3. Demirayak B, Altintas $\mathrm{O}$, Agir $\mathrm{H}$, Alagoz S. Medial Rectus Muscle Injuries after Functional Endoscopic Sinus Surgery. Turk J Ophthalmol 2015; 45(4): 175-8.

4. Huang CM, Meyer DR, Patrinely JR et al. Medial rectus muscle injuries associated with functional endoscopic sinus surgery: characterization and management. Ophthal Plast Reconstr Surg 2003; 19(1): 25-37. 
5. lieva K, Evens PA, Tassignon MJ, Salu P. Ophthalmic complications after functional endoscopic sinus surgery (FESS). Bull Soc Belge Ophtalmol 2008; (308): 9-13.

6. Martinez Del Pero M, Philpott C. A useful tool - systematic checklist for evaluating sinus scans. Clin Otolaryngol 2012; 37(1): 82-4.

7. Sohn JH, Hong SD, Kim JH et al. Extraocular muscle injury during endoscopic sinus surgery: a series of 10 cases at a single center. Rhinology 2014; 52(3): 238-45.
8. Thacker NM, Velez FG, Demer JL, Wang $\mathrm{MB}$, Rosenbaum AL. Extraocular muscle damage associated with endoscopic sinus surgery: an ophthalmology perspective. Am J Rhinol 2005; 19(4): 400-5.

FURTHER READING

Q1: Please check Figure 5.5 caption for corrections.

Q2: Please provide intext citation for Table 5.1.

Q3: Please provide the references to be included in Further Reading. 
